\title{
Vom Diskurs zur Erzählung. Möglichkeiten einer politikwissenschaftlichen Narrativanalyse
}

\author{
Frank Gadinger / Sebastian Jarzebski / Taylan Yildiz
}

From discourse to narration. The promise of narrative analysis in political science

Abstract: Interpretive political science scholars argue that language does not only describe social realities but also serves as a medium of their construction. This minimal consensus, however, leaves one crucial question open. What are the techniques on which this construction of the world is based and how do these rhetorical devices bring about their performative effects? In this article we argue that narratives shape social praxis through the process of narration and, in doing so, enable the political dimension of language. A dialogue between political science and literary studies opens a promising avenue for such a research perspective. We introduce the basic narratology of Albrecht Koschorke to develop a methodology for researching political narratives. To illustrate a narrative analysis and to reflect on its methodological difficulties, we examine different narratives about the riots in the UK in 2011.

Keywords: Narratives, narratology, rhetoric, interpretative research methodology, Albrecht Koschorke

Schlagwörter: Narrative, Erzähltheorie, Rhetorik, Interpretative Politikforschung, Albrecht Koschorke

„Geschichten ereignen sich nicht, Geschichten werden erzählt. “ Christoph Ransmayr (2012, S. 5)

\section{Einleitung: Erzählte Politik'}

Weil Ereignisse nie für sich sprechen, sind wir stets in Geschichten verstrickt. Diese in Wilhelm Schapps „Philosophie der Geschichten“ ([1953] 2012, 1959) formulierte Einsicht schlägt sich neuerdings auch in der sozialwissenschaftlichen Forschungspraxis nieder (u. a. Fisher 1987; Roe 1994; Czarniawska 2004; McCloskey 1998). Das hat nicht zuletzt mit dem Erfolg konstruktivistischer Theorien zu tun und mit der damit einhergehenden Akzeptanz ihrer Kernaussage, dass soziale Realitäten nicht objektiv gegeben sind, sondern einer sprachlichen

1 Für hilfreiche Anmerkungen und Kommentare danken wir den beiden anonymen Gutachter_ innen sowie der PVS-Redaktion. Unser Dank gilt weiter Christian Bueger, Wilhelm Hofmann, Patrick Hohlweck, Claus Leggewie, Daniel Mertens, Holger Niemann, Dirk Peters und Elena Sondermann, die frühere Versionen dieses Aufsatzes gelesen und uns wertvolle Hinweise gegeben haben. Daneben möchten wir uns bei Karl-Rudolf Korte und der „Forschungsgruppe Regieren“ an der NRW School of Governance für die frühe gedankliche und institutionelle Unterstützung des Forschungsprojekts Politische Narrative bedanken. 
Konstruktionsarbeit unterliegen und entgegen noch so restriktiver Bemühungen immer wieder zur Disposition gestellt werden können. Allerdings wäre, wie Albrecht Koschorke (2012, S. 10, Hervorhebung im Original) hierzu anmerkt, noch zu klären, wie die Annahme von „der unhintergehbaren Sprachlichkeit des menschlichen Weltzugangs durch ein Modell der narrativen Organisation dieses Bezuges ergänzt und präzisiert“ werden kann. Im vorliegenden Artikel schließen wir uns seinem Erklärungsversuch an und prüfen dabei, was eine solche Erweiterung politikwissenschaftlich leisten kann: Was bedeutet es, wenn die Erzählung als Medium nicht nur für das menschliche Miteinander, sondern auch für die Politik als konstitutiv erachtet wird?

Folgt man diesem Anliegen, wird man mit zwei politikwissenschaftlichen Verwendungen des Narrativbegriffs konfrontiert, die beide - dies sei vorweggenommen - nur Teilantworten auf die von Koschorke aufgeworfene Frage liefern und damit das analytische Potenzial des Begriffs keineswegs ausschöpfen. Dies ist erstens ein handlungstheoretischer Gebrauch, in dem die komplexitätsreduzierende Wirkung von Narrativen betont wird. Hier verbinden sich eine Reihe innovativer Ansätze aus der interpretativen Policy- (Schön u. Rein 1994; Hajer 1995; Stone [1988] 2002; Viehöver 2006) und Organisationsforschung (Fenton u. Langley 2011; Czarniawska 1997) mit kognitionswissenschaftlichen Studien (Polkinghorne 1988; Bruner 1990) zu der Annahme, dass in schwer fassbaren Situationen Gewissheit letztlich nur auf dem Wege der erzählerischen Deutung der Handlungsprobleme hergestellt werden könne und dieser Vorgang nicht unerheblich für die Verhandlung strategischer Politikoptionen sei. Wenn also auch die Politik stets in Geschichten verstrickt ist, dann nur deshalb, weil politische Akteure unter den Bedingungen begrenzter kognitiver Kapazitäten nicht anders mit der Komplexität der Welt umgehen können.

Im Gegensatz zu dieser Gebrauchsweise lässt sich besonders in den kritischen Ansätzen der Internationalen Beziehungen (IB) eine eher strukturalistische Verwendung des Narrativbegriffs erkennen, die an das Konzept der vorgestellten Gemeinschaften von Benedict Anderson erinnert. Mit dem Narrativbegriff werden hier nicht individuelle, sondern kollektive Sinnmuster gemeint, die jenseits des mentalen Speichers in sogenannten „Erzählgemeinschaften“ zum Ausdruck kommen (Müller-Funk 2008, S. 251; Eder 2009). Zwar liegt auch hier der Fokus auf den Mechanismen der Komplexitätsreduktion, die jetzt aber im Bereich des Diskursiven, d. h. der unterschwelligen Artikulation kollektiver Gewissheiten, verortet werden. Hier geht es weniger um die Frage, wie sich politische Subjekte als handlungsfähige Akteure konstituieren, sondern darum, wie das Politische vor Partikularismen bewahrt und das stets drohende Konfliktpotenzial auf ein verfahrensmäßig regelbares Maß beschränkt werden kann (u. a. Barnett 1999). Gemeinschaftsstiftende Erzählungen sind zwar auch individuell bedeutend, lassen sich aber insofern den kulturellen Gedächtnissen zuordnen, als in ihnen nur das präsent ist, „was den Einzelnen nicht als Einzelnen, sondern als Angehörigen des je zur Rede stehenden Kollektivs [...] betrifft" (Münkler 1996, S. 123). Demnach sind die Handelnden also nie oder zumindest in den seltensten Fällen völlig allein in Geschichten verstrickt, sondern fast immer nur in Relation mit anderen (Wendt 1992, S. 397), ob dies nun Freunde sind, mit denen das Erzählen und Wiederer- 
zählen von kulturell geschlossenen Räumen geteilt wird oder eben Feinde, von denen man sich moralisch und territorial abzugrenzen versucht (Ringmar 1996; Yildiz 2014).

Beide Konzeptionen liefern interessante Perspektiven auf die politische Bindungskraft von Narrativen, werden aber jeweils zu einseitig formuliert, als dass das analytische Potenzial eines solch gehaltvollen Begriffs adäquat umrissen werden könnte. Dies wird etwa daran deutlich, dass der Narrativbegriff auf beiden Seiten noch zu sehr der Konkurrenz seiner dort bereits etablierten Alternativkonstrukte ausgesetzt ist. So erscheint es nahezu unmöglich, den Begriff von mentalistischen Kategorien wie frames, scripts, arguments, ideas oder belief systems abzugrenzen oder einen klaren Unterschied zu strukturalistischen Begriffen wie kollektive Mythen, Identitäten, Weltbilder, Ideologien oder Diskursen herzustellen. Dabei ist ein um den Narrativbegriff gebildetes eigenes Forschungsprofil vielversprechend. Denn anders als die Alternativkonstrukte, die noch zu sehr einer starren Vorstellung von Kulturformen verpflichtet sind, stellt der Erzählbegriff verräumlichende Beschreibungsweisen zur Verfügung, die „Transformationen aller Art und Größenordnung “ (Koschorke 2012, S. 137) ebenso registrierbar machen wie Überschneidungen oder Verästelungen von kulturellen Praktiken. Für ein Studium politischer Prozessdynamiken ist eine auf den Erzählbegriff rekurrierende Forschungsperspektive deshalb besonders gut geeignet.

Mit dem homo narrans (Koschorke 2012, S. 9-12; Barthes 1988, S. 102-142; Fisher 1987, S. 216) wird damit ein entscheidender Perspektivwechsel auf das Soziale/Politische vollzogen; weg von der Anwendung sozialer Regeln auf den sprachlichen Umgang mit Kontingenzerfahrungen. Dabei ist ihm Kontingenz kein Ärgernis, sondern eine Aufgabe, die er auch jenseits vorgegebener Handlungsregeln meistern kann. Anders als die Personen in gängigen Handlungsmodellen ist er imstande, seiner Befähigung, zu den Geschehnissen Ja oder Nein sagen zu können, auch dann zu folgen, wenn ihm rationale Ziel- und Mittelvorgaben (wie beim homo oeconomicus) ebenso fehlen wie eine halbwegs überzeugende Vorstellung vom regelkonformen Verhalten (wie beim homo sociologicus). Der homo narrans ist in der Lage, im Modus der kulturellen Improvisation symbolische Machtkämpfe auszutragen und darüber zu streiten, ob ein „bestimmter Konflikt moralisch, rechtlich oder politisch zu lösen" ist (Koschorke 2008, S. 552-553). Das bedeutet freilich nicht, dass sich der erzählende Mensch nicht auch strategisch oder im Sinne seiner an ihn herangetragenen Erwartungen verhalten könnte. Es bedeutet nur, dass er sich nicht endgültig auf äußerliche Handlungsformeln festlegen lässt, diese stets kritisch hinterfragen kann und selbst dann handlungsfähig bleibt, wenn er sich bewusst oder unbewusst gegen die Imperative gewohnter Handlungslogiken wendet. Mit der Kraft kulturell belastbarer Erzählungen kann er gerade unter den für politische Prozesse typischen Bedingungen der Kontingenz Gewissheit erzeugen und dazu beitragen, dass über bestehende Funktionsgrenzen und definitorische Hoheiten hinweg sozialer Sinn ausgehandelt und kollektive Handlungsfähigkeit hergestellt werden kann (Koschorke 2012, S. 39-40). Der Vorschlag eines erzählerisch orientierten Handlungsbegriffs fügt sich im Übrigen nahtlos in die gegenwärtigen Diskussionen darüber ein, wie dem kreativen Handlungsvermögen im Begriff von agency ein angemessener analytischer Stellenwert 
zugewiesen werden könne (Franke u. Weber 2013), wie gängige Handlungslogiken kritisch zu prüfen seien (Kornprobst 2011) und wie die Fähigkeit der Akteure, in situativen Momenten moralische Urteile zu fällen, auch politikwissenschaftlich beachtet werden könne (Hanrieder 2011).

Der Beitrag zielt damit darauf ab, das Feld der kulturwissenschaftlichen Politologie (Nullmeier 2004) erzähltheoretisch zu erweitern. Er gliedert sich in drei Teile: Zunächst werden wir in Kapitel 2 die konzeptionellen Grundlagen eines narrativen Politikverständnisses entwickeln. Dazu werden wir neben der Erzähltheorie auch interpretative Studien aufgreifen, die im Umfeld der Policy-Forschung und der neueren Organisationsforschung stehen. Die daraus gewonnene Heuristik wird dann in Kapitel 3 mit einem konkreten Fall konfrontiert; mit der politischen Bewältigung der gewaltsamen Proteste in England 2011. Der Fall dient einer ersten vorsichtigen Illustration des Narrativansatzes, der einerseits zeigen soll, wie das Erzählen in interpretativ umkämpften Zonen politisch relevant, ja sogar entscheidend wird. Andererseits soll uns die empirische Arbeit aber auch in die Lage versetzen, die operativen Schwierigkeiten einer Analyse politischer Narrative besser einschätzen zu können. Im Fazit gehen wir dann auf die Möglichkeiten und Grenzen einer Narrativanalyse ein. Im Ergebnis lässt sich feststellen, dass der Erzählbegriff trotz typischer Einführungsprobleme produktiv für die interpretative Politikforschung ist. Denn er eröffnet einen differenzierten Einblick in die sprachlichen Techniken des Schließens politischer Möglichkeitsräume und lässt damit das komplexe Wechselspiel von Ratlosigkeit und politischer Gewissheit gut erkennen. Die zukünftige Herausforderung für eine Narrativanalyse besteht aber in der theoretischen Verfeinerung des Ansatzes und im methodischen Umgang mit der Polyphonie, der Vielstimmigkeit von Erzählungen.

\section{Konzeptionelle Grundlagen: Erzählerische Möglichkeiten}

Den Narrativbegriff in eine operationalisierbare Definition bringen zu wollen, ist kein leichtes Unterfangen. Denn als Medium entzieht sich der Begriff jeder Substanzialisierung, ist modus operandi wie opus operatum zugleich (Viehöver 2006, S. 181) und durchkreuzt damit jede Dichotomie, die sich zwischen dem Realen und Fiktionalen, dem Mythos und Logos schiebt. ${ }^{2}$

Für gewöhnlich wäre dies Grund genug, sich anderen Begrifflichkeiten zuzuwenden. Aber seit Anthony Giddens' Rekapitulation der Akteur-Struktur-Problematik und dem interdisziplinären Erfolg des linguistic turns kommt man nun auch in der Politikwissenschaft kaum mehr umhin, die gängigen Spaltungen der Episteme kritisch zu prüfen und sich ein zumindest rudimentäres Verständnis von der Performativität der Sprache anzueignen. Es lässt sich jedenfalls kaum bestreiten, dass Sprache nicht nur die zu bezeichnenden Sachverhalte und Objekte abbil-

2 Für die Begriffsunterscheidungen zwischen Geschichte, Erzählung und Narration lässt sich keine abschließende Definition finden, die sich in den Wissenschaften durchgesetzt hat. Vielfach werden die Begriffe Geschichte (Story) und Narrativ synonym verwendet (Polkinghorne 1988, S. 14; kritisch hierzu Gabriel 2000, S. 5). Ähnlich verhält es sich mit der Begriffsunterscheidung zwischen Narrativ und Narration, wobei das Narrativ stärker auf das Produkt des Erzählens abhebt (Ryan 2007, S. 22-24) und die Narration eher den Prozess des Erzählens betont (Abbott 2007, S. 39). 
det, sondern umgekehrt, dass sich das Bezeichnete immer auch mittels der ordnenden Kraft der Sprache gestaltet (Rorty 1992, S. 23-24) und dazu beiträgt, dass sich zwischen Ereignis und Wahrnehmung stets „unablässige Anverwandlungen“ (Koschorke 2012, S. 24) vollziehen. Unserer Ansicht nach bietet es sich an, solche Anverwandlungen dem Einsatz erzählerischer Techniken zuzuschreiben. So jedenfalls fasst Barbara Czarniawska (2004, S. 3-6) den Gebrauch des Begriffs als enacted narrative zusammen und Koschorke (2012, S. 62, Hervorhebung im Original) stimmt mit ihr darin überein, dass es schließlich gar keine Ereignisse per se geben könne, diese vielmehr immer nur „Rohmaterial erzählerischen Interesses und Erzeugnisse der narrativen Aufmerksamkeitsführung “ zugleich seien. Wer die Hoheit über das Erzählen erringe, könne schließlich darüber befinden, „welche Geschehnisse überhaupt in die gesellschaftliche Semiosis Eingang finden und tatsächlich Konsequenzen nach sich ziehen“ (Koschorke 2012, S. 62). Für eine Wissenschaft, die sich für politische Interventionen interessiert und die Gestaltungsfähigkeit des Sozialen zu ihrer disziplinären Voraussetzung hat, ist ein solch erzähltheoretisches Sprachverständnis deshalb elementar. Wie sonst könnte die Geburt politischer Reformprojekte und ihre Wanderung in die gesellschaftlichen Verhältnisse nachvollzogen werden, ohne einer rationalistischen Verkürzung von Politik und politischem Handeln zu erliegen?

Das Erzählen ist also immer dort im Spiel, wo sozial Bedeutsames verhandelt wird. Es gleicht einem großen Forum, das „divergenten Stilhaltungen, Sprechweisen, Redepositionen und Fokalisierungen" (Koschorke 2012, S. 19-20) Raum zur Begegnung bietet. Den Rednern etwa eröffnet das Erzählen die Möglichkeit, sich an die Voreinstellungen und Befindlichkeiten auch jenes Auditoriums anzuschließen, die sich außerhalb ihres gesellschaftlichen Bereiches befinden und keinen $\mathrm{Zu}$ gang zu ihrem Spezialwissen finden. Und die Zuhörer versetzt es in die Lage, das Gesagte zu extrapolieren und den Rednern auch dann folgen zu können, wenn ihnen nicht immer alles kognitiv durchdringbar ist - etwa darüber, was nun der Fall ist, welche Konsequenzen drohen, wer dafür die Verantwortung trägt und wie die Politik angesichts solcher Situationen programmiert werden sollte (Hajer 2010, S. 273; Stone [1988] 2002, S. 138). Dem Erzählen obliegt also besonders in Spannungslagen die Aufgabe, einzelne Deutungsschritte so aufeinander abzustimmen, dass kollektives Problemverstehen und -handeln möglich wird. Dieser Aufgabe werden narrative Konstruktionen insofern gerecht, „als sie in ihrer Suche nach Plausibilitäten wenig wählerisch sind [und] auf synkretistische Weise alle verfügbaren Evidenzen“ zusammenziehen (Koschorke 2012, S. 238) und den „Erfolg oder das Scheitern von Wahrheitsansprüchen [...] weniger von empirischer Verifikation als von der inneren Logik und rhetorischen Überzeugungskraft der Erzählung “ (Somers 2012, S. 280) abhängig machen.

Der Narrativbegriff ordnet sich damit in ein Verständnis von Politik ein, das nicht auf materielle Verteilungskonflikte beschränkt ist, sondern ihre Einbettung in die allgemeineren Kämpfe um Artikulationschancen im Fokus hat. Allerdings werden diese Kämpfe nicht aus der Perspektive kampffertiger Akteure rekonstruiert, da theoretisch vorgefertigte Akteursmodelle die kreativen, umstrittenen und oftmals destruktiven Formierungsprozesse realer, um Deutungssicherheit und -macht bemühter Akteure in konkreten Handlungssituationen ausblenden. Daher 
bedienen wir uns der erzähltheoretisch angeleiteten Prämisse, dass organisierte Akteure - seien es Völker, Staaten, Parteien, Regierungen, Verbände, Bewegungen oder auch Individuen - grundsätzlich als Prozesse zu verstehen sind und ihre zentralen Formierungsimpulse durch Narrative im Sinne eines organizational storytelling erhalten (Czarniawska 1997, S. 26-29; Gabriel 2000, S. 19-25; Boje 1991, S. 106-111). Eine Erzähltheorie kann damit zu einem besseren politikwissenschaftlichen Verständnis darüber beitragen, wie konfrontative Sprachstrategien in Prozesse der kollektiven Selbstverständigung übergehen und wie auf diese Weise schließlich eine emergente Erzählordnung entsteht, in deren Sinnbezügen sich eine kollektiv verbindliche Entscheidung einrichten und mit Legitimität versorgen kann. Um einen solchen Vorgang auch empirisch rekonstruieren zu können, werden nun die zentralen Erzähltechniken und -ressourcen vorgestellt: Das emplotment, verstanden als kausale Modellierung loser Erfahrungen (2.1), die Plot-Muster (2.2) und Tropen (2.3), mittels derer die kausal konfigurierten Geschehnisse rhetorisch abgesichert werden. Das Kapitel schließt mit einer übersetzungssoziologischen Konzeption erzählerischer Vorgänge ab (2.4) und unternimmt darin den Versuch, die Allgemeine Erzähltheorie von Koschorke mit einer programmatischen Unterscheidung von Akteur und Subjekt zu erweitern.

\subsection{Emplotment: Sinnbildung durch kausale Modellierung von Erfahrung}

Der Narrativbegriff ist aus der antiken Rhetorik überliefert. Er beschreibt dort jenen Teil einer Rede, in dem „ein als Handlungsablauf faßbares Geschehen“ mitgeteilt wird (Knape 2003, S. 98). Dabei wird die narrative „Schilderung des Sachverhalts" (Göttert 2009, S. 33), in dem ort-zeit-verschiedene Erfahrungen zusammengeführt werden, als eine dem Argument vorausgehende Aktivität aufgefasst, was insofern zutreffend ist, als ohne sinnvollen Bezug zu einer Vorstellung von den Geschehnissen keine Argumentation vollzogen werden kann. Die moderne Literaturwissenschaft hat nun das Studium dieser Zusammenhänge geerbt. Aber als „leading discipline in the study of narrative“ (Meuter 2009, S. 243) versteht sie Narrative nicht mehr nur als Teil einer Rede, sondern erhebt sie in den Rang eines disziplinären Oberbegriffes. Damit kehrt sich das Verhältnis zwischen Rede und Erzählung um. Das Narrativ ist jetzt nicht mehr Mittel der Rede, sondern die Rede eine unter vielen Möglichkeiten bestehende Form des Umgangs mit Erzählstoffen. Der Hinweis darauf, dass ein „als Handlungsablauf faßbares Geschehen“ nur als Narrativ mitteilbar ist und dieser Vorgang als konstitutiv für das Argumentieren gedacht werden sollte, bleibt aber im Erbe nach wie vor erhalten.

Zur Beschreibung dieser Erzähltechnik hat die moderne Literaturwissenschaft den Begriff des emplotment entwickelt. Kognitionspsychologisch handelt es sich dabei um ein zentrales Verfahren der kognitiven Komplexitätsreduktion (Rath 2011, S. 16-25), weil es zum einen die Sequenzierung der Ereignisse auf eine Weise ermöglicht, die den Erfahrungshorizont der Akteure nicht überstrapaziert und zum anderen eine so vollzogene Ordnung der Eindrücke auch über Funktionsbereiche hinweg vermittelt werden kann (Polkinghorne 1988, S. 11). Damit ergibt sich eine erste begriffliche Präzisierung. Nur solche Sprachkonstrukte können als Narrative verstanden werden, die im Gegensatz zu bloßen Chroni- 
ken, Listen, Nachrichten und Typologien singuläre Vorfälle in einen sinnvollen Ereigniszusammenhang bringen und so den Eindrücken eine historische Tiefe und Kohärenz verleihen (White 1987, S. 4). Margaret R. Somers (1994, S. 616) weist an dieser Stelle darauf hin, dass die narrative Entlastung des Menschen meist als kausale Festlegung erfolgt und dass es sich bei Narrativen um raum-zeit-bezogene „constellations of relationships (connected parts)“ handelt, die durch ein sogenanntes causal emplotment konstituiert werden (vgl. auch Tilly 2008, S. 64-65). Die Annahme, dass keine Sequenz von Ereignissen beschrieben werden kann, ohne ihr eine zumindest vermutungsweise angedeutete Kausalität zu unterlegen, liegt hier jedenfalls nahe. So wird mittels des emplotments aus einem bloßen Neben- und Nacheinander ein sinnhaft geordnetes und handlungsstiftendes „Wegeneinander" (Koschorke 2012, S. 75).

Der narrative Kausalitätsbegriff grenzt sich dabei von einem wissenschaftstheoretischen Verständnis kausaler Zusammenhänge ab. Weder will er überprüfbare Ursache-Wirkungs-Beziehungen zum Ausdruck bringen, noch läuft er auf die Erwartung hinaus, dass sich die erzählten Geschehnisse unter universellen Gesetzmäßigkeiten subsumieren ließen. Vielmehr bezieht sich der Begriff des causal emplotment auf die handlungsleitenden, sich teilweise sogar widersprechenden Kausalitätspostulate historisch situierter Akteure, also auf jene situativen Zurichtungen der Kausalität, die im Kontext konkreter Projekte mobilisiert werden, aber nicht notwendigerweise zutreffen müssen (Bruner 1990, S. 44). Auch wenn das emplotment damit grundsätzlich kontingent ist und stets von der Existenz konkurrierender Wahrheiten (Rimmon-Kenan 2006, S. 12) bedroht wird, können die darin enthaltenen Kausalvorstellungen im weiteren Verlauf der Ereignisse transgressive Effekte erzeugen. Denn ist ein Plot einmal etabliert, liegt die Zurechnung von Begebenheiten auf Akteure nicht weit. Und diese Zurechnung vollzieht sich insbesondere durch narrative Sprechpraktiken. So wird die Ursache zum Verursacher und die Lösung zum Erlöser und beide laden auf unterschiedliche Weise zur Solidarisierung oder gar Nachahmung ein. Kurzum: Das emplotment ist folgenreich, weil es Identitäten und Optionen perspektiviert und Realitäten dem Druck moralischer Urteile unterzieht (White 1987, S. 14).

Hier bricht ein erzähltheoretischer Narrativbegriff aus der kognitionspsychologischen Enge, weil er die Performativität von Narrativen nicht nur daran erkennbar macht, dass einzelnen Akteuren eine imaginäre Außenperspektive ermöglicht wird, sondern auch zeigt, dass Narrative im „Medium sozial geteilten Wissens gedeihen “ (Koschorke 2012, S. 37) und für den Erzählverlauf nicht unwesentlich ist, wie die Rezipienten die Geschichte auffüllen, sie tentativ ergänzen und ihre Kohärenz zu stärken versuchen (Koschorke 2012, S. 76). Erzählungen wandern also nicht unverändert durch soziale Funktionsgrenzen. Vielmehr wird ihnen stets etwas Neues hinzuerzählt, etwas abgerungen oder eine folgenreiche Variation eingeführt. Ein weiteres Problem kognitionspsychologischer Bestimmungen liegt in ihrer alleinigen Fixierung auf Vorgänge der Komplexitäts- oder Kontingenzreduktion. Mit Narrativen wird zwar die sprachliche Konstruktion kausaler Beziehungen meist relativ einfach gehalten (Tilly 2008, S. 65, 72) und oftmals wird diese Einfachheit auch beharrlich verteidigt (Wagenaar 2011, S. 216). Aber hinsichtlich ihres Gegenstandes sind Narrative insofern ontologisch indifferent, als das Erzäh- 
len „ebensogut in den Dienst des Abbaus von Sinnbezügen gestellt werden [kann], etwa durch die Demontage von hegemonialen Sinnzwängen." In einer Vielzahl von Erzählungen, so Koschorke (2012, S. 11), „wird Kontingenz keineswegs gebannt, sondern geradezu heraufbeschworen."

\subsection{Plot-Muster und Erzählweisen}

Das Erzählen von Kausalitäten verfährt nicht im Stil wissenschaftlicher Beweisführung, gleicht eher dem Verfahren der Abduktion und ist durch den „hypothetischen Rückschluss von einer überraschenden Einzeltatsache auf eine ihr möglicherweise zugrundeliegende Regel“ (Koschorke 2012, S. 65) gekennzeichnet. Allerdings lassen sich so völlig unterschiedliche Verknüpfungen herstellen. Im Modus der Abduktion ist die kausale Komposition vereinzelter Eindrücke auf völlig unterschiedlichen Wegen erreichbar. Hier stellt sich nun die Frage, wie sich eine bestimmte Erzählversion gegen konkurrierende Darstellungen durchsetzen und ihre eigene Kontingenz im Verlauf der kollektiven Sinnverhandlung verschleiern kann. Die Antwort liegt - grob gesprochen - im Potenzial einer Erzählung, „soziale Erfahrungen und kulturelle Gedächtnisbestände“ so zu aktivieren, dass neue Vorkommnisse, so überraschend sie auch sein mögen, in die Plot-Struktur einer jeweiligen Erzählung eingebaut werden können und dadurch den „Anschein natürlicher Evidenz" erhalten (Koschorke 2012, S. 245). Um diese Dimension des Erzählens erfassen zu können, hat die Literaturwissenschaft das Repertoire kulturell belastbarer Plot-Muster zu identifizieren versucht. Prominent wurde insbesondere die Einteilung Hayden Whites, der in Anschluss an Northrop Frye mit der Romanze, der Satire, der Komödie und der Tragödie vier grundlegende Prinzipien bestimmt hatte, wie Kausalität kulturell anschlussfähig modelliert werden kann (White 2008, S. 21-25; siehe hierzu auch Viehöver 2012, S. 84-85; Czarniawska 2004, S. 20-21). Die Romanze beispielsweise ermöglicht es, normative Ansprüche zu fokussieren und den Sieg des Guten über das Böse zu betonen. Angelpunkt einer Romanze ist stets die „Überschreitung der Erfahrungswelt“ (White 2008, S. 22) durch den Helden, der nur so im Kampf gegen das Böse den drohenden Niedergang abwenden kann. Die Satire hingegen kehrt dieses Befreiungsmuster um und betont das Gefühl von Ausweglosigkeit, das in Kontingenzerfahrungen spürbar wird. Durch komödiantische Plot-Muster wiederum werden die Eindrücke so zu einem zusammenhängenden Geschehen konfiguriert, dass Kausalität sich in den Dienst der „Versöhnung der in Gesellschaft und Natur wirkenden Kräfte“ (White 2008, S. 23) stellen kann. Von Versöhnungen erzählen Tragödien eher nicht. Ähnlich wie in der Satire werden auch hier ebenso langfristige, unabänderliche Bedingungen geschaffen. Diese sind in der Tragödie allerdings ,düster und bedeuten eher, dass sich die Menschen in die Bedingungen ihres mühseligen irdischen Daseins schicken müssen“ (White 2008, S. 23).

Diese Unterscheidungen wurden nun vielfach aufgegriffen, um etwa narrative Praktiken in Organisationen zu studieren (Gabriel 2000, S. 84-85; Boje 1991). So hat sich in der Politikwissenschaft die Annahme formieren können, dass politische Kontroversen durch ein begrenztes Set kausaler Erzählmuster strukturiert werden und auch in der Politik Rollen und Ereignisse narrativ konfiguriert und zueinander 
in Beziehung gesetzt werden. Dies fängt, wie Deborah Stone ([1988] 2002, S. 138) beobachten konnte, bereits mit der Definition von Policy-Problemen an:

"Definitions of policy problems usually have narrative structure; that is, they are stories with a beginning, a middle, and an end, involving some change or transformation. They have heroes and villains and innocent victims, and they pit the forces of evil against the forces of good. The story line in policy writing is often hidden, but one should not be thwarted by the surface details from searching for the underlying story. Often what appears as conflict over details is really disagreement about the fundamental story."

Bei den fundamentalen Geschichten, auf deren Relevanz Stone hier verweist, handelt es sich im Kern um die von White als Plot-Muster bezeichneten Tiefenstrukturen von Erzählungen. Auch Stone ([1988] 2002, S. 138-143) führt schließlich mit diesem Verweis vier typische Erzählmuster ein, die sie für den Verlauf politischer Kontroversen für prägend hält: Die Erzählung des Niedergangs (story of decline), die Erzählung des verhinderten Fortschritts (story of stymied progress), die Erzählung des vermeintlichen Wandels (change-is-only-an-illusion-story) und die Erzählung der Hilflosigkeit und Kontrolle (story of helplessness and control). All diese Muster sind moralisch keinesfalls neutral, sondern führen jeweils eigene Urteile mit (Wagenaar 2011, S. 213). Auch wirken sie nicht deterministisch, so als ob wir nicht anders könnten, als den Tiefenstrukturen unserer Geschichten zu folgen. Vielmehr sollten wir diese Muster im Sinne der Allgemeinen Erzähltheorie Koschorkes als Foren begreifen, in denen sich Erzähler und Rezipienten begegnen und darüber streiten können, wie eine entgleiste Welt (wieder) in Ordnung zu bringen sei. Eine solche Kategorisierung kann zwar die Gesamtheit aller denkbaren PlotMuster sicherlich nicht umfassen, zumal sich die Liste durch solch hybride Kategorien wie das Sozialdrama oder die Tragikomödie sinnvoll erweitern ließe. Sie kann jedoch einer Studie zu politischen Narrativen als heuristisches Rüstzeug dienen und die empirische Rekonstruktion politischer Narrative durchaus erleichtern.

\subsection{Erzählerische Instrumente: Tropen}

Die Verknüpfung loser Ereignisse in ein kausal zusammenhängendes Geschehnis ist ohne die kreativen Momente des Sprachgebrauchs undenkbar. Neben PlotMustern operieren Narrative auch mit rhetorischen Figuren, die die jeweilige Synthesis der Ereignisse begrifflich verdichten können und das, was dem physischen Auge entgeht, in ein sprachlich mitteilbares Bild kleiden. Diese Figuren werden in der Literaturwissenschaft als Tropen bezeichnet und sollen hier in Abgrenzung zu Stone ([1988] 2002, S. 137-162) nicht als ein den Narrativen gleichgestelltes Sprachkonstrukt, sondern als elementares Mittel des Erzählens verstanden werden. Die wichtigsten dieser rhetorischen Figuren sind die Metapher und die Metonymie (Culler 2002, S. 105; Koschorke et al. 2007, S. 55-64), wobei andere Autoren auch die Synekdoche und die Ironie in diesem Zusammenhang diskutieren 
(Burke 1941, S. 421; White 2008, S. 50-57)3. Die Metonymie etwa macht Sinn dadurch übertragbar, indem sie Begriffe, die derselben semantischen Ebene angehören und in einer Beziehung der Kontiguität stehen, zum jeweiligen Stellvertreter erheben lässt; wenn etwa der Effekt zur Ursache und die Ursache zum Verursacher wird und sich die Eindrücke so ordnen, dass die Herstellung politischer Verantwortlichkeiten möglich wird. In der Politik liegt eine Metonymie etwa dann vor, wenn über einen Einzelfall eine größere Problematik angesprochen werden soll, wenn beispielsweise in der Diskussion um Integrationsprobleme und Schulpolitik die Verwendung des Begriffs Neukölln ganz bestimmte Assoziationen weckt. Handelt es sich um ein Spiel mit den semantischen Relationen der Kontiguität, so spricht man spezieller von einer Synekdoche, wenn etwa die Hand den Arbeiter symbolisiert oder das Gehirn den Intellektuellen.

Insbesondere das bildhafte Sprechen in Analogien aber, das durch den Einsatz von Metaphern möglich wird, kann dazu beitragen, unverständlichen Vorfällen einen Sinn abzugewinnen und unter den Bedingungen des Zeitmangels kommunikativ anschlussfähige Handlungsoptionen und -begründungen $\mathrm{zu}$ generieren (Lakoff u. Johnson 1998). Gerade im Gebrauch von Metaphern dokumentiert sich auf faszinierende Weise, dass rhetorische Mittel Einfluss auf politische Wirklichkeitskonstruktionen nehmen können und Wirklichkeit nicht nur durch das konstruiert wird, was gesagt wird, sondern auch durch die Art und Weise, wie es gesagt wird (Hülsse 2003, S. 212). Rainer Hülsse hat diese von Lakoff und Johnson formulierte Überlegung mit einer Studie zu den innereuropäischen Auseinandersetzungen mit dem EU-Beitrittsantrag der Türkei unterstützen können. Gerade die Metapher einer an die Tür Europas anklopfenden Türkei, so Hülsse, habe sich nicht nur auf die Beitrittsfrage selbst, sondern auch auf die EU-Identitätsdebatte ausgewirkt. Noch grundsätzlicher haben Koschorke et al. (2007, S. 63) die performative Kraft von Metaphern betont. Am Beispiel der Entwicklung des neuzeitlichen Flächenstaates konnte er zeigen, dass seine soziale Konstruktion ohne die performative Kraft der Körperschaftsmetaphorik nicht adäquat nachvollzogen werden kann. Selbst in nicht-westlichen Herrschaftsverbänden konnte sich die Metapher als äußerst folgenträchtig erweisen (Yildiz 2012). So gesehen besteht die Aufgabe von Metaphern nicht etwa darin, „eine präzise Bezeichnung einer realen Situation zu erzeugen, für deren Beschreibung die Worte fehlen [...], sondern diese Realität durch selektive Repräsentationen in eine imaginäre Bildökonomie zu übersetzen “, um so kontingente Realitäten beherrschbar machen zu können (Mackenthun 2010, S. 123).

3 Die sprachtheoretische Diskussion über die Abgrenzung von Synekdoche und Metonymie soll hier nicht weiter vertieft werden. White behandelt diese Problematik ausführlich in den Anmerkungen seiner Metahistory (White 2008, S. 573-576). Culler hingegen weist darauf hin, dass die „Metapher als grundlegendes Phänomen der Sprache wie der Imagination ausgewiesen " wurde (Culler 2002, S. 105). Entscheidend für unseren Zusammenhang ist die Wirkung rhetorischer Mittel in Narrativen. 


\subsection{Fluide Erzähleinheiten: Vielstimmigkeit und Übersetzung}

Die bisherigen Überlegungen verdeutlichen, dass eine kognitionswissenschaftliche Sicht auf Narrative anregend für Sozialwissenschaftler_innen ist, aber insgesamt zu kurz greift, weil sie nicht den für Narrative erforderlichen Blick auf Prozesse des kollektiven Verstehens bietet. Dafür wäre eine kulturwissenschaftlich sensible Perspektive vonnöten, die das Erzählen nicht auf mentale Ressourcen und Aktivitäten allein zurückführt, sondern ihren Weltbezug und ihre Intertextualität mitdenkt. Denn die soziale Wirkungskraft von Narrativen bliebe unvollständig rekonstruiert, beschränkte man sich auf die innere Struktur von Erzählungen und ließe die Frage nach ihren strukturellen Kontexten außer Acht. Allerdings sollte auch in diesem Bemühen ein Determinismus vermieden werden, der sich nun in Form der theoretischen Konstruktion subjektunabhängiger Sinn- und Zeichenwelten in die Konzeption wieder einschleichen könnte (Reckwitz 2010, S. 15-46).

Um dieser Aufgabe gerecht zu werden, nimmt Koschorke - wenn man es genauer betrachtet - nicht den Akteur, sondern das Subjekt zum Ausgangspunkt seiner Allgemeinen Erzähltheorie (Koschorke 2012, S. 84-90). Denn im Gegensatz zum Akteur - in der englischsprachigen Debatte wird diese Verschiebung meist mit dem Begriff des agents anstelle des actors zum Ausdruck gebracht - steht das Subjekt in einem weitaus fließenderen Verhältnis zu seinen inneren und äußeren Spannungen und ermöglicht damit größere Einblicke in die Möglichkeitsräume des Handelns. Während der Akteur darauf beharrt, diese Zugänge bereits erfolgreich verschlossen und so einen Ort geschaffen zu haben, in dem er seine Präferenzen oder einschlägige Normen weitestgehend selbstbestimmt reflektieren kann, erweist sich das Subjekt als „wanderndes, disperses und vielfach fragmentiertes Wesen “ (Koschorke 2012, S. 84). Und weil ihm letzte Gewissheiten fehlen, pflegt er einen viel offeneren Umgang mit seinen Kontingenzerfahrungen. Und da sich Subjekte eben nicht durch festgeschriebene Handlungsregeln definieren, sondern durch die Fähigkeit zum artistischen Spiel mit den Restbeständen ihrer Ordnungen, erscheint ihnen das Fragmentiert-Sein nicht als Mangelzustand, sondern als Möglichkeit, aus heterogenen Materialien neue Strukturen zu erschaffen, statt sich in bereits strukturierten Einheiten einzurichten, so wie es der Akteur zu tun gewohnt ist. Daher droht ihm im Umgang mit den Brüchen und Kontingenzen seines Daseins nicht gleich die Existenzfrage. Denn wo der Akteur keine Handlungsregeln anwenden kann und sich völlig überfordert der Ausweglosigkeit ausgesetzt sieht, findet das Subjekt die Bedingungen seines schöpferischen Potenzials wieder (Joas 1996, S. 15). So erweist sich das, was aus der Perspektive herkömmlicher Handlungstypen zunächst als Nachteil erscheint, als notwendige Bedingung eines kritischen Denk- und Schaffensprozesses.

Es ist nun keineswegs so, dass einzelne Individuen als Gesamtwesen in die eine oder andere Kategorie zugeordnet werden könnten, so als wäre es nur wenigen Avantgardisten gestattet, in die Existenzform eines Subjekts einzukehren und sich seiner Fähigkeiten zu bemächtigen, während den anderen nichts anderes übrig bliebe, als ihr kreatives Potenzial auf die Befolgung vorab festgelegter Auslegungsund Handlungsroutinen zu beschränken. Vielmehr wird damit eine Spannung beschrieben, die grundsätzlich alle Individuen betrifft, die sich, gewollt oder unge- 
wollt, mit den Kontingenzen und Brüchen ihres Daseins auseinandersetzen. Die praktischen und kritischen Kompetenzen gewöhnlicher Akteure werden dadurch substanziell aufgewertet (Boltanski 2010, S. 50-56).

Mit der Konzeption des homo narrans wird ein solches Subjektverständnis nun so ausformuliert, dass jene erzählerisch-kreativen Techniken fokussierbar werden, die sich in einem funktional noch unberührten Raum „des Dazwischen oder Davor" (Koschorke 2008, S. 552) ereignen und für die Konstruktion sozialer Tatsachen (im Sinne getaner Sachen) als elementar erachtet werden können. Der Vorteil eines solch fluiden Subjektverständnisses liegt eben darin, dass es den zwischenmenschlichen Raum nicht auf das funktional Durchdrungene beschränkt, sondern in seiner Polyphonie mitzudenken und als jenen Ort zu begreifen erlaubt, in dem sich die Konstitution und Übertragung von Sinn jenseits systemischer Zwänge vollziehen kann.

Dieser Raum lässt sich konzeptionell in zweierlei Hinsicht verorten. Erstens als eine „mächtige dritte Größe“, die sich zwischen die Erzählenden und Rezipienten schiebt und ko-präsente Kommunikationsakte organisiert (Koschorke 2012, S. 87); und zweitens als ein Raum, der zeitlich zurückliegenden Erfahrungen und Texten Relevanz zu verleihen imstande ist. Theoretisch gesprochen ist die Erzählung also jener Stoff, den die Übertragung von Sinn im funktional noch undifferenzierten Raum in Anspruch nehmen muss, ob nun in synchroner oder diachroner Weise (siehe Abb. 1).

\section{Abb. 1: Erzählen im Prozess der Übersetzung (eigene Darstellung in Anlehnung an Koschorke 2012, S. 88)}

Außenweltbezug | Intertexualität

Erzähler/Rezipient Verhältnis

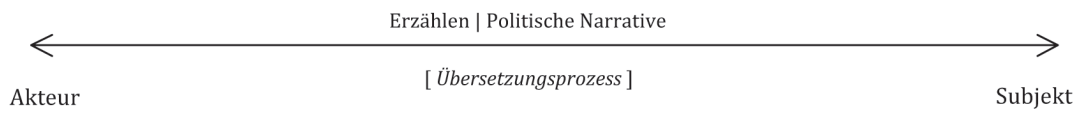

Neuerdings wird ein solcher narrative approach auch in der Organisationsforschung genutzt (Czarniawska 1997; Fenton u. Langley 2011) und mithilfe der Akteur-Netzwerk-Theorie bzw. der Soziologie der Übersetzung (Latour 2007) in eine positive Untersuchungsheuristik überführt. ${ }^{4}$ Denn die empirische Analyse von Organisationen hat gezeigt, dass selbst funktional gut durchdrungene Organisationen stets prozesshaft sind, also auch dann noch mit Räumen des „Dazwischen und Davor“ auskommen müssen, wenn sie sich längst etabliert haben. Das führt dazu, dass Organisationen fortlaufend konstitutive Prozesse durchlaufen müssen und die Performativität des Erzählens, die sich in solchen Prozessen ausdrückt, jenem Vorgang gleicht, der in der französischen Soziologie unter dem Begriff der Übersetzung verhandelt wird: die stetige Verknüpfung völlig heterogener Entitäten zu einer handlungsfähigen Einheit, dem Aktanten. Der Begriff ist der Strukturellen

4 Dies liegt insofern nahe, als Autoren wie Michel Callon und Bruno Latour ihrerseits von literaturwissenschaftlichen Erzähltheorien - insbesondere von Algirdas Julien Greimas' - inspiriert sind und sich methodologisch an ihrer „Bewegungsfreiheit“ orientieren (Latour 2007, S. 96). 
Semantik Algirdas Julien Greimas' (1971) entlehnt und behandelt analog zum oben beschriebenen Subjektbegriff die Tatsache, dass selbst überschaubare Handlungen und Ereignisse nie auf einen Handelnden allein zurückgerechnet werden können, sondern allenfalls auf eine komplexe Relation von Handelnden, die - und das macht ihre Komplexität aus - nicht notwendigerweise auf menschliche und ko-präsente Wesen beschränkt sein muss. Dabei fällt auf, dass die sprachliche Beschreibung derartiger Relationen stets die Form einer Erzählung annimmt und die Tätigkeit, die damit geleistet wird, sich als Übersetzung heterogener Ziele, Interessen und Vorrichtungen verstehen lässt. Denn mit einer Erzählung lässt sich ziemlich kohärent das ausdrücken, „was andere sagen und wünschen, warum sie auf diese eine Weise handeln, wie sie sich mit anderen verbinden“. Und am vorläufigen Ende einer erzählerischen Komposition der Welt, so Michel Callon (2006, S. 196), „sind nur noch im Gleichklang sprechende Stimmen zu hören.“ Und sofern die Übersetzung gelingt, wird die „Problematisierung, die nur eine einfache Idee war, [...] in Mobilisierung transformiert. [...] Die Übersetzung geht weiter, aber das Gleichgewicht wurde modifiziert" (Callon 2006, S. 169-170).

Die Parallelen zur Erzähltheorie sind unübersehbar. Gerade der Gedanke, dass sich vorgestellte Gleichgewichtszustände mittels einer Erzählung transformieren, die heterogene Entitäten zu einem einheitlich Willen übersetzen kann, bringt die Nähe der Erzähltheorie zur Akteur-Netzwerk-Theorie gut zum Ausdruck. Folgt man diesem Bündnis zwischen Erzähl- und Akteur-Netzwerk-Theorie, wird deutlich, warum eine Erzählung immer nur temporär zum Abschluss gelangen kann. Denn stets droht die Übersetzung zu scheitern oder von den Entitäten, die übersetzt werden, eingeholt zu werden. Sei es, weil sich das zu Übersetzende widersetzt, weil es etwas Widerspenstiges hinzuerzählt, oder aber, weil sich die Objekte und Artefakte der Außenwelt verändern und diese Veränderung einen erneuten Erzählvorgang anregt. Gerade für eine Politikwissenschaft, die sich normativ wie empirisch mit der spannungsgeladenen Herstellung eines kollektiven Willens und Makrosubjekts beschäftigt, lässt ein solcher Erzählbegriff höchst signifikante Kontexte erschließen. ${ }^{5}$ Denn schließlich liefert das „Repertoire der Übersetzung “ auch eine Erklärung dafür, „wie einige das Recht erhalten, die vielen von ihnen mobilisierten stillen Akteure der sozialen und natürlichen Welt zu repräsentieren und für sie zu sprechen“ (Callon 2006, S. 170).

5 An dieser Stelle macht Koschorke auf eine bemerkenswerte Sinnverwandtschaft der Erzähltheorie mit den politikwissenschaftlichen Imperiumstheorien aufmerksam, wie sie insbesondere Michael Mann und Herfried Münkler vorgelegt haben. Siehe dazu insbesondere den vierten Grund, den Koschorke für eine Neubeschäftigung mit der Kultursemiotik Jurij Lotmans anführt (Koschorke 2012, S. 125-128). Dort heißt es etwa: „Denn die historische Schaukelbewegung, die nach Michael Mann den Takt der Imperien schlägt, bildet ein herrschaftstechnisches Äquivalent zu den Zeichenprozessen, die Lotman zufolge an der Peripherie von Semiosphären wirksam sind“ (Koschorke 2012, S. 128). Ähnlich ließe sich der Anarchiebegriff der Internationalen Beziehungen als liminale Zone reformulieren und so kulturwissenschaftlich zugänglich machen. 


\section{Die UK-Riots im narrativen Untersuchungsdesign}

Es stellt sich nun die Frage, wie sich vor dem Hintergrund dieser Methodologie eine Narrativanalyse forschungspraktisch bewerkstelligen lässt. Ihr Ziel kann zwar aufgezeigt werden: Die Narrativanalyse sollte sich nicht in einer bloßen Beschreibung der Erzählungen erschöpfen, sondern vielmehr darauf abzielen, ihre politischen Effekte aufzuzeigen. Auch dürfte es ihr nicht darum gehen, Erzählungen möglichst präzise voneinander abzugrenzen, also die Erzählstoffe typologisch abzurunden. Sie müsste sich auf die wechselseitige Konstitution unterschiedlicher Erzählmomente fokussieren und das zu identifizieren versuchen, was man eine sich hegemonialisierende Erzählung nennen könnte. Abgesehen von diesen allgemeinen Anforderungen, ist die Methodik narrativer Analysen aber noch klärungsbedürftig. Zwar lässt sich diese Aufgabe an dieser Stelle nicht abschließend bearbeiten, bedürfte sie doch einer vertieften Anwendung in empirischen Studien. Aber einige forschungspraktische Überlegungen und empirische Illustrationen lassen sich in diesem Bemühen durchaus anführen.

Zwei Vorschläge scheinen uns wichtig, um die Narrativanalyse an ihre eigenen methodischen Schwierigkeiten heranführen zu können: Erstens gehen wir davon aus, dass sich eine narrative Methode zwar an bewährten diskursanalytischen $\mathrm{Zu}$ gängen orientieren kann (Mishler 1995; Riessman 1993), doch letztlich, wie Czarniawska (2004, S. 88) völlig zu Recht betont, darauf hinauslaufen muss, einen Text danach zu befragen, was dieser tut und nicht - wie in Diskursanalysen üblich -, was er aussagt und mit welchen versteckten Annahmen er operiert (Czarniawska 2004, S. 97). Zwar ist das Hintergrundwissen einzelner Aussageereignisse auch hier wichtig. Aber es geht in viel stärkerem Maße um ihre Einbettung in soziale Praktiken und darum, wie das Erzählte gerade in diskursiv unbestimmten Räumen des „Dazwischen und Davor“ den Makel des Partikularen ablegen und ein kollektives Gewissheitsempfinden für rätselhafte Ereignisse vermitteln kann. Ein diskursiver Raum zeichnet sich durch die Wirksamkeit gesetzter Sinnroutinen aus, „die mit den Körpern, die sie enthält, keine andere Beziehung eingeht, als ihnen ihre Position in einem von jedem beliebigen Punkt aus zu errichtenden Koordinatenkreuz zuzuweisen“ (Koschorke 2012, S. 112). Das Erzählen dagegen gleicht eher einem Beziehungsraum oder einer liminalen Zone, die von der Unruhe der Objektbewegungen erfasst wird und sich an die Formen und Körper anschmiegt, die vergeblich nach ihrer diskursiven Bestimmung suchen. Dies ist die „Zone fließender Bedeutungen, Zugehörigkeiten und Identitäten, in der gesellschaftliche Strukturschemata nicht mehr greifen, vielmehr auflösende Kräfte am Werk sind“ (Koschorke 2012, S. 115).

Unser zweiter Vorschlag zur Entwicklung einer positiven Heuristik bezieht sich auf die Frage, wie man in eine Analyse politischer Narrative einsteigen kann, wenn die darin verknüpften Einheiten letztlich so einheitlich nicht sind: Wenn Struktur nur als übersetzte Struktur studiert werden kann und die Übersetzung stets andere Raumwelten zu erzeugen vermag; wenn sich Erzähler und Rezipienten erst im Erzählverlauf formieren, dann muss eine empirische Narrativanalyse anders als auf der Grundlage dieser Untersuchungskategorien eingeführt werden (vgl. hierzu auch Gadinger et al. 2014a). Mit Somers (2012, S. 288) gehen wir 
hier davon aus, dass politische Narrative innerhalb sozialer Krisenerfahrungen entstehen und der „Kampf um kommunikatives Kapital [...] mit allen Mitteln einer sheißen Semiotik ' geführt wird" (Koschorke 2012, S. 136). Gerade in Situationen höchster Kontingenz und Ratlosigkeit, so die Annahme, die auch in der Tradition des Amerikanischen Pragmatismus (John Dewey) steht, tritt die performative Kraft von Narrativen aus dem Schatten institutioneller Routinen hervor und wird dadurch sichtbar. Deshalb sollte eine Narrativanalyse in interpretativ umkämpften Zonen ihren Anlauf nehmen und dort nach den narrativen Techniken Ausschau halten, die in Kapitel 2 skizziert wurden.

Als geeignetes Beispiel zur Illustration einer politikwissenschaftlichen Narrativanalyse dient uns hier jener Fall, der am 4. August 2011 mit dem Tod Mark Duggans seinen Ausgang nahm und sich auf rätselhafte Weise zu dem entwickelte, was in der globalen Öffentlichkeit als UK-Riots kontrovers diskutiert wurde. Eine zunächst friedliche Demonstration gegen örtliche Polizeigewalt verwandelte sich binnen weniger Tage zu einer Gewaltwelle, die mehrere britische Vororte in ihren Bann zog und politische Akteure, Sozialwissenschaftler_innen und Medienvertreter_innen gleichermaßen vor das Problem stellte, die Geschehnisse so zu deuten, dass eine annähernd schlüssige und überzeugende Erklärung öffentlich verhandelbar wurde (Morrell et al. 2011, S. 13-24). An diesem Fall lässt sich gut nachvollziehen, wie aus zunächst verschieden motivierten Plausibilisierungsversuchen eine emergente Erzählordnung entsteht, auf deren Grundlage sich eine politische Strategie formiert, die als legitim erachtet wird und sich vor kritischen Einwänden behaupten kann. Bei der interpretativen Rekonstruktion zeigt sich, dass sich die Kontroverse nicht als Wettstreit zwischen progressiven und konservativen oder rechten und linken Narrativen strukturiert. Bei genauerem narrativ-fokussiertem Hinsehen wird vielmehr deutlich, dass sich die zunächst selektiv begründeten Erzählbeiträge im weiteren Verlauf der Debatte wechselseitig konstituieren und gemeinsam an der Hervorbringung einer öffentlichen Erzählung arbeiten, die sich an den Problemhorizonten der jeweiligen (polyphonen) Erzähler abarbeiten und erst dadurch eine hegemoniale Stellung erlangen kann. Das bedeutet nicht, dass einzelne Erzählbeiträge bewusst aufeinander abgestimmt werden. Es bedeutet nur, dass sie sich wechselseitig zur Voraussetzung haben. In dieser funktionalen Verschränkung ist schließlich die Möglichkeit eines stillen Kompromisses angelegt, auf den sich die weiteren Erzählbeiträge rückbeziehen können, wenn über politische Partikularismen hinweg Legitimität beansprucht werden soll. Damit stellt sich eine Narrativanalyse zwar weitaus komplexer dar, als eine in Argumenten und Diskursen aufgelöste Lagerperspektive, die dem "Ineinandergreifen strukturierender und entstrukturierender Tendenzen" (Koschorke 2012, S. 119, Hervorhebung im Original) hilflos ausgesetzt ist. Sie ermöglicht aber, dass wir einen näheren Einblick in die Dynamik von Diskursen und Argumenten und damit in die sprachliche Konstruktion von Politik überhaupt erhalten können.

Da die Rekonstruktion einer wechselseitigen Konstitution von Argumenten/ Artikulationen zu Narrativen detaillierte prozessanalytische Schritte erfordert, die unsere illustrativen Absichten weit übersteigt, haben wir uns dazu entschieden, die im Verlauf der Ereignisse vorgelegten Deutungsangebote als Narrative zu re- 
konstruieren ${ }^{6}$ und diese Rekonstruktion so zu gestalten, dass ihre Bezüge zueinander sichtbar werden.

\subsection{Das regulative Narrativ: Die Romanze von der broken society}

Das regulative Narrativ formierte sich im politischen Feld und trug die Züge einer Romanze, die keine Grauzonen, sondern nur Dichotomien kennt und die soziale Synthesis in Varianten des Guten und Bösen spaltet. Im ausgewählten Fall fand diese Spaltung ihren Höhepunkt in einer Rede von David Cameron (2011). Sie lief dort über ein emplotment, das die kollektive Gewalt als nicht entschuldbaren Ausdruck der moralischen Schwäche einer gang culture präsentierte, die allein durch die Aktivierung der Rechtschaffenden aufzuhalten war. Der Öffentlichkeit wurde diese Verknüpfung unter anderem mit einem Video einprägsam, in dem Jugendliche sich am Rande der Proteste gegenseitig berauben (TheKoshyar 2011). Ihren moralischen Ausgleich fand diese Veranschaulichung von „purer Kriminalität" in der symbolischen Kraft des Besens, mit dem jene Anwohner zu Rechtsschaffenden erklärt wurden, die mit „brooms to clean up“ die Schäden der Gewaltnächte zu bereinigen versuchten (McDermott 2011). In beiden Szenen wurden einzelne Details ikonisch derart isoliert, dass weitläufige Handlungskontexte abrufbar (Koschorke 2012, S. 72) und zur kausalen Verknüpfung nutzbar gemacht werden konnten.

Zunächst wurden die Szenen nur vereinzelt wahrgenommen, konnten dann aber mit Camerons Einführung der Metapher einer broken society mit einem regulativen Plot-Muster operieren und sich entsprechend erweitern. Zwei narrative Übersetzungspraktiken waren dafür elementar: Erstens konnte mithilfe der Bruchmetapher die szenisch verdichteten Urteile in strukturierte Aussagen über die Ursachen und Folgen der Gewalt überführt werden. Wie kontrovers die kausale Komposition der Ereignisse auch erfolgte, die Metapher des Bruches machte es möglich, die Gegenwart als Wendepunkt zu begreifen und über sinnvolle Optionen zu streiten. Die Romanze jedenfalls führte den gesellschaftlichen Bruch auf die Tat einer moralisch degenerierten Jugend zurück, die ermittelt und zur Rechenschaft gezogen werden müsse. Für die Lösung bot die Romanze eine schlagkräftige Figur: den Helden, der innerhalb des metaphorischen Spiels mit dem Bruch als ein Wesen vorstellbar wurde, das die Rolle des Kehrenden ausfüllt und die soziale Ordnung wiederherstellt.

Zweitens macht die Romanze deutlich, dass einzelne Erzählpraktiken sich erst dann ausbreiten, wenn sie auch mit Plausibilitäten arbeiten, die sie selbst nicht

6 Eine Narrativanalyse folgt einem weiten, kulturwissenschaftlich inspirierten Textverständnis, das auch vermeintliche low data als gleichwertige Quellen ansieht. Zur empirischen Analyse der konkurrierenden Narrativen haben wir uns neben offiziellen Verlautbarungen der politischen Akteure deswegen auch mediale Darstellungen der UK-Riots, wie Youtube-Videos, Fernsehinterviews und Talkshows sowie Bilder und wissenschaftliche Studien angesehen, die in diesem Kontext auch als Narrationen zu sehen sind. Dieser Argumentation folgend, könnte man auch den hier vorliegenden Text als wissenschaftliches Narrativ entlarven. Dieser Kritik kann man sehr offensiv begegnen, da die Universalität des Erzählens als Modus der Vermittlung auch vor der Wissenschaft nicht Halt macht, zumal wenn man sich im Bereich der Textwissenschaft bewegt. 
erschaffen haben. So wurde in Camerons Rede etwa die Dramaturgie einer systemkritischen Tragödie, die von strukturell bedingten und unaufhaltsam voranschreitenden Niedergängen berichtete, für die Legitimation eines Helden nutzbar gemacht, der die politische Kontrolle innerhalb bestehender Macht- und Herrschaftstechniken zurückzuerobern versprach. Unterstützt wurde diese romantische Wendung der Tragödie, die besonders in den Medien dominant werden konnte, durch die Verwendung eines medizinischen Vokabulars. Denn als Krankheitsdiagnose (Cameron 2011) vergegenwärtigt, wurde es möglich, den Bedarf operativer Maßnahmen zu unterstreichen und den Protest zu pathologisieren, sodass die Folgen unverhältnismäßiger Polizeigewalt als hinnehmbare Kollateralschäden begriffen werden konnten.

Mit diesen diskursiven Verschiebungen ließ sich jegliches Verständnis für die Jugendlichen marginalisieren und im Gegenzug Legitimität für grundsätzlich autoritäre Praktiken erzeugen. Denn während die Eskalation der Gewalt in der kritischen Deutung als tragische Folge eines nationalstaatlich kaum mehr zu bändigenden globalen Finanzkapitalismus präsentiert wurde und damit ein gewisses Maß an Verständnis für den Unmut einer perspektivlosen Jugend zuließ (Malik 2011), wurden die Ereignisse mit der Einführung der Bruchmetapher zum Kampf von Gut (we/us) und Böse (thugs) erklärt (Cameron 2011). Erzähler, die sich diesem Narrativ widersetzten und darauf verwiesen, dass die Gründe der Ausschreitungen auch in einer zunehmenden sozialen Ungleichheit lägen (Natcen1 2012) wurden dadurch entkräftet. Der Vorteil einer Romanze besteht offenbar darin, dass sie aus vagen Strukturen Rollen und Handlungsabläufe generiert, die bestehende Stereotype aufrufen können. Wenngleich Cameron (2011) mehrmals betonte, dass „these riots were not about race“, so zeigen die in Anspruch genommenen Bilder doch zugleich, dass die Spaltung der britischen Gesellschaft in Gut und Böse durchaus rassische Komponenten mitführt. Jedenfalls kennt das Bild der Rechtsschaffenden kaum people of colour, wogegen die broomers allein der solventeren weißen Mittelschicht anzugehören scheinen. Und umgekehrt agiert die Ikonografie der Protestbewegung hauptsächlich in der Farbe schwarz.

\subsection{Das appellative Narrativ: Die komödiantische Rettung}

Dass erzählerische Verknüpfungsleistungen kontingent sind, dokumentiert sich zunächst in der Rolle, die durch die appellative Erzählung in der öffentlichen Auseinandersetzung erfüllt wurde. Sie folgte den Erzählstrukturen einer Komödie und richtete sich damit auf jene erzählerisch unbestimmte Grauzone, die die Romanze bewusst ausgeklammert hatte. Damit konnte sie einerseits die Verunsicherung aufgreifen, die sich auf den Straßen artikulierte. Andererseits kam sie damit aber auch in die Lage, jene regulativen Erzählungen mitzubestimmen, die sich auf der Seite staatlicher Ordnungskräfte etablierten. Getrieben von der Hoffnung auf Versöhnung (White 2008, S. 23) erkannte sie die Existenz gesellschaftlicher Probleme an, verurteilte jedoch zugleich das Ausmaß der Ausschreitungen. So forderten etliche Erzählstimmen die Regierung auf, ihre Helden in Stellung zu bringen und die Sicherheit der Bürger und ihres Eigentums zu gewährleisten. Zugleich forderten diese Stimmen aber auch, dass solche Bemühungen durch langfristig angelegte 
Maßnahmen begleitet werden müssten und dies letztlich nur durch strukturelle Verbesserungen in der Sozialpolitik erreicht werden könne.

Mit der komödiantischen Besetzung der diskursiven Grauzone dreht sich das Verhältnis von Gut und Böse nicht gänzlich, aber teilweise um. Da dieser erzählerische Raum durchlässig ist, kann sie die Seite des Guten mit weiteren Rollen aufstocken, insbesondere mit solchen, die die Versöhnung vorantreiben. Neben Polizisten, Staatsanwälten und Richtern treten damit Sozialarbeiter und insbesondere jugendliche Multiplikatoren als Helden auf. Und das Böse besteht nicht mehr ausschließlich aus Protestlern, sondern erreicht nun auch jene politischen Eliten, die für Sozialabbau verantwortlich gemacht werden können. Mit dieser Verschränkung und Ausdifferenzierung der Rollen ließ sich das konfrontative Potenzial dichotomer Beschreibungen teilweise bewältigen. Zwar wird auch hier die Tragödie überwunden und an den Erfolg des Helden geglaubt. Doch die Komödie traut den einfachen Mitteln der Romanze - insbesondere ihren Moralisierungen - nicht und verlangt, dass auch der Held, da er stets zu scheitern droht, seine Aufgaben und Grenzen kritisch zu reflektieren hat. Eine zentrale narrative Technik dieser Wendung liegt allerdings nicht nur in der Ausdifferenzierung der Rollen, sondern auch in der Wahl des Anfangs und Endpunktes der Erzählung. Es scheint gerade so, als ob die Ausweitung des zeitlichen Horizonts dazu führt, dass die Erzählung authentischer wird, und zwar insofern als dadurch eine Reihe unbeachteter Kontingenzerfahrungen registrierbar wird.

Auch dieses Narrativ hatte einprägsame Bilder zu bieten, etwa den erzählerisch wirkungsmächtigen Auftritt der Heroine of Hackney, die sich in einem zur Online-Berühmtheit gelangten Handyvideo über die Krawalle echauffiert (XItwigyIX 2011; Gardham 2011). Ihre Stimme ist insofern charakteristisch für eine Komödie, als sie die Kontroverse in die Proteste verlagert und dort mit großer Authentizität an die Vernunft der Protestierenden appellieren kann. Aber auch innerhalb der türkischstämmigen Community Londons ließen sich ähnliche Stimmen vernehmen. Die Nachrichten füllten sich mit Videos, in denen Händler ihre Geschäfte vor Plünderungen schützten, den Einsatz der Polizei verlangten, aber dennoch die Situation dazu nutzten, Probleme der sozialen Verteilungsproblematik anzusprechen (DutchTurkish 2011; Gaylipoo 2011). Selbst die vom Cabinet Office in Auftrag gegebene Studie „The August riots in England: Understanding the involvement of young people" lässt sich als Stimme des appellativen Narrativs lesen, weil hier mit wissenschaftlichem Optimismus und nah an der Politik eine Reihe korrektiver Aspekte formuliert wird, die sich der regulativen Erzählung entziehen (Morrell et al. 2011, S. 4).

\subsection{Das anti-regulative Narrativ: „Fight The Power Not The Powerless“}

Dem auf gesellschaftliche Einträchtigkeit ausgerichteten appellativen Narrativ tritt nun die in Gestalt einer Satire gekleidete anti-regulative Erzählung entgegen. Die Kritik richtet sich in erster Linie gegen die Austeritäts- und Spending-Cuts-Politik der Regierung Cameron, die - so die kausale Zusammenführung der Ereignisse zwangsläufig in einen gewaltsamen Protest münden musste. In die Rolle des Protagonisten schlüpft der an seiner Entfaltung gehinderte Protestler und das System 
wird als sein wahrer Gegenspieler entlarvt. Erzähler des anti-regulativen Narrativs arbeiten zwar ähnlich wie die appellative Erzählung mit der Ausdehnung des zeitlichen Horizonts. Doch dies dient nicht der Versöhnung im Hier und Jetzt, sondern dem Anstoß eines möglichst grundsätzlichen Politikwechsels. Daher sind die Erzähler auch darum bemüht, eine größtmöglich differenzierte Analyse der Situation zu kommunizieren und beharren gleichsam auf die „endgültigen Unzulänglichkeiten der Weltdeutungen “ (White 2008, S. 25) konkurrierender Narrative. Der gesellschaftliche Fortschritt sei durch das politische Handeln behindert und ein Ausbruch aus den bestehenden Verhältnissen legitim und notwendig. Nur ein brachialer Akt könne schließlich die gesellschaftliche Wiedereingliederung der Ausgegrenzten veranlassen, da andere, friedlichere Mittel nicht mehr ausreichen würden.

Matt Corr, Sprecher der 99\% Campaign, tritt hier als exemplarische Stimme auf: „There are policy-decisions, that could have made differently. [...] Bankers come away with the same kind of looting, just in a different context" (NatCen1 2012). Er sieht zwei Systeme der Gerechtigkeit am Werk, eines, das auf einflussreiche Personen zugeschnitten sei und ein weiteres, das für die Ausgegrenzten gelte und sie mit einer falsch fokussierten Sozialarbeit konfrontiere und das Problem der Ungleichheit und Arbeitslosigkeit systematisch ausklammere. Hier findet sich erneut die Metapher der broken society, die von Cameron etabliert wurde. Jedoch wird sie in einen konträren Plot überführt. Ist sie bei Cameron noch Zeichen für eine moralische Spaltung in Gut und Böse, in Rechtschaffende und Kriminelle, so steht sie hier für Ausgrenzung und soziale Ungleichheit. Im öffentlichen Diskurs nehmen die Aktanten dieses Narrativs die Rolle des Anwalts der Armen ein, die Handlungsempfehlungen an die Politik nicht mit dem Ziel einer Versöhnung richten, sondern als Anstoß für einen grundlegenden Politikwechsel.

Neben der 99\% Campaign wurde diese kausale Zurichtung der Ereignisse von einer Studie mit dem Titel „Reading the Riots“ unterstützt, die der Guardian gemeinsam mit der London School of Economics herausgegeben hatte und dadurch die Vielzahl erzählerischer Stimmen institutionell verankern konnte. Entgegen der Behauptung des regulativen Narrativs kommt die Studie zur Erkenntnis, dass weder Gangs die treibende Kraft waren, noch die Aktivierung durch soziale Medien, wie Twitter, Facebook und BlackBerry, sondern eine Polizeigewalt, die im Verbund mit Diskriminierung, mangelndem Respekt, Arbeitslosigkeit und dem Opportunismus des Moments auftritt (Roberts 2011). Die Parole „Fight The Power Not The Powerless“ (genesiselijahtv 2011) fasst diesen Tenor griffig zusammen.

\subsection{Das konfrontative Narrativ: Die Tragödie der „Best Protests Ever!“}

Schließlich lässt sich noch eine weitere Erzählung identifizieren, die sich am ehesten der Tragödie (White 2008, S. 23) oder einer story of decline (Stone [1988] 2002, S. 138-143) zuordnen lässt. Auch sie hat Erzählfragmente zu ihrer Voraussetzung, die sie selbst nicht erschaffen hat, insbesondere solche, die dem anti-regulativen Muster entsprechen. Aber sie verlagert die Perspektive auf die Mikroebene, bietet individuelle Geschichten an und artikuliert sich in erster Linie durch die Personen, die aktiv an den Ausschreitungen Teil hatten. Sie sehen ihre Gewalt im Kontext eines Protestes gegen ein System, welches sie unterdrückt und ausgrenzt. 
Sie haben gewissermaßen zum letzten Mittel gegriffen, welches ihnen noch geblieben ist, um aus der realen Katastrophe und den damit einhergehenden individuellen Tragödien zu entfliehen. In dieser Erzählung ist der Held eine gescheiterte Figur, die in einem letzten Kraftakt ihren Untergang billigend in Kauf nimmt, um dem Zuschauer die wahre Struktur und die wirklichen Gesetze der Gesellschaft zu offenbaren (White 2008, S. 23). Das konfrontative Narrativ will aufrütteln, deutet die Krise so, dass der gegenwärtige Protest als letzte Stufe der Eskalation erscheint. Den Erzählern geht es darum, klarzustellen, dass ihre Duldsamkeit Grenzen erreicht hat. Was Stone ([1988] 2002, S. 138) als Gefühl der Ungerechtigkeit eines „something has to be done“ beschreibt, zeigt sich auch in den Rechtfertigungen Jugendlicher aus Tottenham, die das konfrontative Narrativ erzählerisch prägen:

A: "The government they took it too far now! People are trying to stand up for themselves"

Q: "Was it a real protest against the system?"

A: "I think that is the best protest that happened, ever happened!" (Notesproducer 2011)

Dabei wird oft Rache als Leitmotiv für erlittene Ungerechtigkeiten angegeben. So berichtet ein Jugendlicher, der einen Shop in Clapham ausraubte, bei dem er sich vergeblich beworben hatte:

"Obviously, if they ain't noticing me from that [die Bewerbung] I mind off for starting it a different way. [...] They don't give us the opportunity for working as hard as you. We can do exactly what you can do. [...] The government just cares for the rich people, they don't care for us. [...] We didn't it for the fun of it, we did it for money to survive in this world. Until we get that, or a little bit of support from the government, it is not gonna stop." (nsotd2 2011)

Auch hier ist das System der Feind und auch hier wird seine Doppelmoral problematisiert. Aber aus der Mikroperspektive wirkt dieser Feind übermächtig. Er entzieht damit jeglichen Glauben an die Vernunft der Gemeinschaft oder an ihre Fähigkeit, mit irgendeiner Maßnahme den schmerzhaft erfahrenen Bruch zu überwinden und die Macht des Feindes zu brechen. Die Erfahrung ist endgültig, weil sich unter dem Druck einer Übermacht jede Ecke verdunkelt und es so scheint, als ob das Gefühl der Machtlosigkeit allein durch ein überhöhtes Maß an Euphorie kompensierbar wird.

\section{Fazit}

Eine erzähltheoretische Perspektive auf politische Zusammenhänge zu formulieren ist - wie alle Erneuerungsbemühungen - durch Offenheit und Ungewissheit gekennzeichnet. Aber gerade darin liegt auch ihr Vorteil. Denn sie erhebt diese elementare Erfahrung des Forschungsprozesses zum Ausgangspunkt ihrer konzeptionellen Entwicklung und nähert sich damit auch dem Wunsch, sich von den gängigen Akteursmodellen zu lösen und die Motivbildungen im Untersuchungsfeld jenseits vordefinierter Machtinteressen und Erwartungen als offene Prozesse 
zu rekonstruieren (Nullmeier 1999, S. 230). Wie unsere Illustration gezeigt hat, lohnt es sich, diesen Versuch vor dem Hintergrund der erzählerischen Konstruktionsarbeit in Krisenmomenten zu unternehmen und möglichst nah an den Ereignissen darauf zu achten, was die Akteure in ihrem kreativen Vermögen und ihrem gesteigerten Artikulationswillen nicht alles unternehmen, um dem Publikum überzeugende Geschichten anzubieten und die mannigfaltigen Orientierungen und Motive innerhalb ihres gesellschaftlichen Kontextes zu vernetzen. Narrativforscher stehen damit vor der keineswegs einfachen Aufgabe, sich einer verworrenen Vielstimmigkeit zu stellen und sich dem Gebot sparsamer Erklärungen bewusst zu entziehen. Erzählungen in der permanenten Übersetzung zwischen erfinderischen Akteuren und temporären Strukturen einzukreisen, erfordert gewaltige methodologische und methodische Anstrengungen, die in einem beginnenden Forschungsprogramm (Gadinger et al. 2014b; Hofmann et al. 2014) bewältigt werden müssen. Dieses steht vor einer Problematik, die Parallelen zur Heisenbergschen Unschärferelation und ihren forschungspragmatischen Schwierigkeiten aufweist: Es scheint unmöglich, den Ort und die Geschwindigkeit der Teilchen in einem Schritt zu bestimmen. Übertragen auf die Narrativanalyse müssen wir stets den Fokus auf den Prozess des Erzählens (die Geschwindigkeit) oder den Gegenstand des Erzählten (den Ort) legen.

Der Rückbezug zur modernen Literatur- und Kulturwissenschaft scheint uns insofern hilfreich, als Politikwissenschaftlern meist gar nicht bewusst ist, dass Erzähltechniken bereits einen entscheidenden Einfluss auf den Prozess der Verbindlichkeitsherstellung ausüben und damit die Konstruktion sozialer Realität wesentlich mitprägen. Dem Fokus auf Argumente und Inhalte bleibt das Wie einer sprachlichen Konstruktionsarbeit meist verschlossen. Schließt man diese Lücke mit dem Narrativbegriff, so unsere These, wird die Arbeit an der Kontingenz ebenso sichtbar wie die Rekonstruktion der sprachlichen Strategien ihrer Bewältigung möglich wird. Die politikwissenschaftliche Beschäftigung mit Narrativen schließt damit weitaus stärker an kultur- und medienwissenschaftliche Perspektiven an, als dies beispielsweise in der mittlerweile fest etablierten Diskursanalyse geschieht. In der Narrativanalyse, wie wir sie in diesem Beitrag mithilfe der Allgemeinen Erzähltheorie Koschorkes und der Policy- und Organisationsforschung entwickelt haben, geht es weniger um das Aufdecken diskursiver Machtverhältnisse oder um die versteckten, stillen Dynamiken diskursiver Verschiebungen, sondern um die Frage nach den sprachlichen Techniken der Legitimitätserzeugung (wobei das Technische hier in einem praxeologischen und medientheoretischen Sinne zu verstehen ist). Die Analyse schließt dabei sowohl Eliten als auch Alltagssubjekte ein, da besonders für Demokratien unterstellt werden kann, dass sie über institutionell abgesicherte Responsivitätsschleifen verfügen und damit vergleichsweise gut auf öffentliche Erzählvorgänge geeicht sind. Das muss jedoch, wie die empirische Illustration zeigt, nicht bedeuten, dass allein die Existenz solcher Mechanismen schon dauerhaft zur demokratischen Qualitätssicherung beitragen könnte. Vielmehr legt die Fallanalyse die demokratietheoretisch bedenkliche These nahe, dass politische Narrative gerade dann bindend werden können, wenn sie mit einfachen Plots operieren und überschaubare Vorstellungen von der Kausalität der Ereignisse erzeugen. Gerade zur Untersuchung unübersichtlicher Krisensi- 
tuationen, wie der globalen Finanzkrise oder EU-Staatsschuldenkrise, bietet sich die Narrativanalyse an, um mitunter herauszufinden, warum es den Akteuren nicht gelingt, ,überzeugende Erzählungen` anzubieten.

Der transdisziplinäre Charakter und die noch weitgehend fehlende methodologische Übertragung auf die Politikwissenschaften sollte nicht als riskante Verwässerung ihrer Forschungsagenda gesehen werden, sondern als Möglichkeit, das konstruktivistische Forschungsprogramm zu erweitern. Die Skepsis gegenüber einem offeneren Zugang zu Narrativen in der Politikwissenschaft liegt sicherlich auch darin begründet, dass die Hinwendung zur Populär- bzw. Alltagskultur als Widerspruch zu den großen universellen Theorien bzw. Erzählungen verstanden werden könnte, wie dies unter poststrukturalistischen Denkern wie Jean-François Lyotard (1986), Jacques Derrida (2000) oder Michel Foucault (1974) zwar üblich ist, aber von manchen Sozialwissenschaftler_innen nach wie vor als Gefahr für die wissenschaftliche Forschungspraxis insgesamt angesehen wird (Patterson u. Monroe 1998, S. 318). Zudem, und dies schwingt beim narrative turn stets mit, sind Narrative als methodologisches Werkzeug unvermeidbar von der Aura der Fiktion umgeben, was vielen positivistisch geprägten Politikwissenschaftler_innen hinsichtlich ihrer eigenen Bewertungsmaßstäbe unangenehm sein dürfte. Was hätte denn Fiktion schon mit Realität zu tun? Die Kritik, dass das Studieren von Narrativen mehr Kunst als Forschung sei, wird zwar häufig geäußert, jedoch wird letztlich selten überzeugend argumentiert, warum Kunst nicht die gleiche Wertschätzung als Wissen zukommen sollte (Rhodes u. Brown 2005, S. 168), wo sich doch das Fiktive nicht selten als historische Triebkraft herausgestellt hat.

\section{Literatur}

Abbott, H. Porter. 2007. Story, Plot and Narration. In The Cambridge Companion to Narrative, Hrsg. David Herman, 39-51. Cambridge: Cambridge University Press.

Barnett, Michael. 1999. Culture, Strategy, and Foreign Policy Change: Israel's Road to Oslo. European Journal of International Relations 5: 5-36.

Barthes, Roland. 1988. Das semiologische Abenteuer. Frankfurt a. M.: Suhrkamp.

Boje, David M. 1991. The Storytelling Organization: A Study of Story Performance in an Office-Supply Firm. Administrative Science Quarterly 36: 106-126.

Boltanski, Luc. 2010. Soziologie und Sozialkritik. Berlin: Suhrkamp.

Bruner, Jerome. 1990. Acts of Meaning. Cambridge: Harvard University Press.

Burke, Kenneth. 1941. Four Master Tropes. The Kenyon Review 3: 421-438.

Callon, Michel. 2006. Einige Elemente der Soziologie der Übersetzung: Die Domestikation der Kammmuscheln und der Fischer der St. Brieuc-Bucht. In ANThology. Ein einführendes Handbuch zur Akteur-Netzwerk-Theorie, Hrsg. Andréa Belliger und David J. Krieger, 135-174. Bielefeld: transcript.

Cameron, David. 2011. Prime Minister's speech on the fightback after the riots. http:// www.number10.gov.uk/news/pms-speech-on-the-fightback-after-the-riots. Zugegriffen: 22.01.2013.

Culler, Jonathan. 2002. Literaturtheorie. Eine kurze Einführung. Stuttgart: Reclam.

Czarniawska, Barbara. 1997. Narrating the Organization. Dramas of Institutional Identity. Chicago: University of Chicago Press. 
Czarniawska, Barbara. 2004. Narratives in Social Science Research. Los Angeles: Sage. Derrida, Jacques. 2000. Die Schrift und die Differenz. Frankfurt a. M.: Suhrkamp.

Eder, Klaus. 2009. A Theory of Collective Identity Making Sense of the Debate on a 'European Identity'. European Journal of Social Theory 12: 427-447.

Fenton, C. und A. Langley. 2011. Strategy as Practice and the Narrative Turn. Organization Studies 32: 1171-1196.

Fisher, Walter R. 1987. Human Communication as Narration: Toward a Philosophy of Reason, Value, and Action. Columbia: University of South Carolina Press.

Foucault, Michel. 1974. Die Ordnung der Dinge. Eine Archäologie der Humanwissenschaften. Frankfurt a. M.: Suhrkamp.

Franke, Ulrich, und Ralph Weber. 2013. At the Papini Hotel. On Pragmatism in the Study of International Relations. European Journal of International Relations 18: 669-691.

Gabriel, Yiannis. 2000. Storytelling in Organizations. Facts, Fictions, and Fantasies. Oxford: Oxford University Press.

Gadinger, Frank, Sebastian Jarzebski, und Taylan Yildiz. 2014a. Politische Narrative. Konturen eines erzähltheoretischen Forschungsprogramms in der Politikwissenschaft. In dies.: Politische Narrative, Wiesbaden: Springer VS (im Erscheinen).

Gadinger, Frank, Sebastian Jarzebski, und Taylan Yildiz (Hrsg.). 2014b. Politische Narrative. Wiesbaden: Springer VS (im Erscheinen).

Gardham, Duncan. 2011. 'Hackney speech woman' revealed to be local jazz singer. http:// www.telegraph.co.uk/news/uknews/crime/8692712/Hackney-speech-woman-revealedto-be-local-jazz-singer.html. Zugegriffen: 23.01.2013.

Göttert, Karl-Heinz. 2009. Einführung in die Rhetorik. Paderborn: Wilhelm Fink Verlag.

Greimas, Algirdas Julien. 1971. Strukturale Semantik. Methodologische Untersuchungen. Braunschweig: Vieweg + Sohn.

Hajer, Maarten A. 1995. The Politics of Environmental Discourse. Ecological Modernization and the Policy Process. Oxford: Oxford University Press.

Hajer, Maarten A. 2010. Argumentative Diskursanalyse. Auf der Suche nach Koalitionen, Praktiken und Bedeutung. In Handbuch sozialwissenschaftliche Diskursanalyse. Band 2: Forschungspraxis, Hrsg. Rainer Keller, Andreas Hirseland, Werner Schneider und Willy Viehöver, 271-298. Wiesbaden: VS Verlag für Sozialwissenschaften.

Hanrieder, Tine. 2011. The False Promise of the Better Argument. International Theory 3: 390-415.

Hofmann, Wilhelm, Judith Renner, und Katja Teich (Hrsg.). 2014. Narrative Formen der Politik, Wiesbaden: Springer VS (im Erscheinen).

Hülsse, Rainer. 2003. Sprache ist mehr als Argumentation. Zur wirklichkeitskonstituierenden Rolle von Metaphern. Zeitschrift für Internationale Beziehungen 10: 211-246.

Joas, Hans. 1996. Die Kreativität des Handelns. Frankfurt a. M.: Suhrkamp.

Knape, Joachim. 2003. Narratio. In Historisches Wörterbuch der Rhetorik. Bd. 6 MustPop, Hrsg. Gert Ueding, 98-106. Tübingen: Niemeyer.

Kornprobst, Markus. 2011. The agent's logics of action: defining and mapping political judgment. International Theory 3: 70-104.

Koschorke, Albrecht. 2008. Codes und Narrative. Überlegungen zur Poetik der funktionalen Differenzierung. In Texte zur Literaturtheorie der Gegenwart, Hrsg. Dorothee Kimmich, Rolf Günter Renner und Bernd Stiegler, 545-558. Stuttgart: Reclam. 
Koschorke, Albrecht. 2012. Wahrheit und Erfindung. Grundzüge einer Allgemeinen Erzähltheorie. Frankfurt a. M.: S. Fischer.

Koschorke, Albrecht, Susanne Lüdemann, Thomas Frank, und Ethel Matala de Mazza. 2007. Der fiktive Staat. Konstruktionen des politischen Körpers in der Geschichte Europas. Frankfurt a. M.: S. Fischer.

Lakoff, George, und Mark Johnson. 1998. Leben in Metaphern. Konstruktion und Gebrauch von Sprachbildern. Heidelberg: Carl Auer.

Latour, Bruno. 2007. Eine neue Soziologie für eine neue Gesellschaft. Frankfurt a. M.: Suhrkamp.

Lyotard, Jean-François. 1986. Das postmoderne Wissen. Ein Bericht. Graz-Wien: Böhlau.

Mackenthun, Gesa. 2010. Von Hybriden und Geisterschiffen. Metaphern im postkolonialen Wissenschaftsdiskurs. In Metaphern in Wissenskulturen, Hrsg. Matthias Junge, 123-139. Wiesbaden: VS Verlag für Sozialwissenschaften.

Malik, Shiv. 2011. UK riots: 'We don't want no trouble. We just want a job'. http://www. guardian.co.uk/uk/2011/aug/12/uk-riots-analysis. Zugegriffen: 22.01.2013.

McCloskey, Deirdre. 1998. The Rhetorics of Economics. Madison: The University of Wisconsin Press.

McDermott, Nicholas, und William Jaffray. 2011. \#Riot Wombles on the march: 200 rioters caused this mayhem...500 offer help to clean up mess. http:/www.dailymail.co.uk/ news/article-2024035/UK-riots-2011-500-Londoners-offer-help-clean-rioters-mess. html. Zugegriffen: 22.01.2013.

Meuter, Norbert. 2009. Narration in Various Disciplines. In Handbook of Narratology, Hrsg. Peter Hühn, John Pier, Wolf Schmid und Jörg Schönert, 242-262. Berlin/New York: De Gruyter.

Mishler, Elliot G. 1995. Models of Narrative Analysis. A Typology. Journal of Narrative and Life History 5: 87-124.

Morrell, Gareth, Sara Scott, Di McNeish, und Stephen Webster. 2011. The August riots in England. Understanding the involvement of young people. London: National Centre for Social Science.

Müller-Funk, Wolfgang. 2008. Die Kultur und ihre Narrative. Wien/New York: Springer.

Münkler, Herfried. 1996. Politische Mythen und Institutionenwandel. Die Anstrengungen der DDR, sich ein eigenes kollektives Gedächtnis zu verschaffen. In Institutionenwandel. Leviathan Sonderheft 16, Hrsg. G. Göhler, 121-142. Opladen: Westdeutscher Verlag.

Nullmeier, Frank. 1999. Interpretative Politikanalyse in der Theoriekonkurrenz. In Interpretation, Konstruktion, Kultur. Ein Paradigmenwechsel in den Sozialwissenschaften, Hrsg. Andreas Reckwitz und Holger Sievert, 219-238. Opladen: Westdeutscher Verlag. Nullmeier, Frank. 2004. Methodenfragen einer kulturwissenschaftlichen Politologie. In Handbuch der Kulturwissenschaften. Bd. 2: Paradigmen und Disziplinen, Hrsg. Friedrich Jäger und Jürgen Straub, 486-501. Stuttgart/Weimar: J.B. Metzler.

Patterson, Molly, und Kristen Renwick Monroe. 1998. Narrative in Political Science. Annual Review of Political Science 1: 315-331.

Polkinghorne, Donald E. 1988. Narrative Knowing and the Human Sciences. Albany: State University of New York Press.

Ransmayr, Christoph. 2012. Atlas eines ängstlichen Mannes. Frankfurt a. M.: Fischer.

Rath, Brigitte. 2011. Narratives Verstehen. Entwurf eines narrativen Schemas. Göttingen: Velbrück. 
Reckwitz, Andreas. 2010. Unscharfe Grenzen. Perspektiven der Kultursoziologie. Bielefeld: transcript.

Rhodes, Carl, und Andrew D. Brown. 2005. Narrative, organizations and research. International Journal of Management Reviews 7: 167-188.

Riessman, Catherine Kohler. 1993. Narrative Analysis. Thousand Oaks: Sage.

Ringmar, Erik. 1996. On the Ontological Status of the State. European Journal of International Relations 2: 439-466.

Rimmon-Kenan, Shlomith. 2006. Concepts of Narrative. In The Travelling Concept of Narrative. Collegium Vol. 1, Hrsg. Matti Hyvärinen, Anu Korhonen, und Juri Mykkänen, 10-19. Helsinki: Helsinki Collegium for Advanced Studies.

Roberts, Dan (Hrsg.). 2011. Reading the Riots. Investigating England's Summer of Disorder. London: Guardian Books.

Roe, Emery. 1994. Narrative Policy Analysis. Theory and Practice. Durham/London: Duke University Press.

Rorty, Richard. 1992. Kontingenz, Ironie und Solidarität. Frankfurt a. M.: Suhrkamp.

Ryan, Marie-Laure. 2007. Toward a definition of narrative. In The Cambridge Companion to Narrative, Hrsg. David Herman, 22-38. Cambridge: Cambridge University Press.

Schapp, Wilhelm. [1953] 2012. In Geschichten verstrickt. Zum Sein von Mensch und Ding. Frankfurt a. M.: Klostermann.

Schapp, Wilhelm. 1959. Philosophie der Geschichten. Frankfurt a. M.: Klostermann.

Schön, Donald E., und Martin Rein. 1994. Frame Reflection. Toward the Resolution of Intractable Policy Controversies. New York: Basic Books.

Somers, Margaret R. 1994. The narrative constitution of identity: A relational and network approach. Theory and Society 23: 605-649.

Somers, Margaret R. 2012. Angst und Schrecken vor der Öffentlichkeit. Der narrative Diskurs der angloamerikanischen citizenship theory. In Erzählungen im Öffentlichen, Hrsg. Markus Arnold, Gert Dressel und Willy Viehöver, 273-309. Wiesbaden: Springer VS.

Stone, Deborah. [1988] 2002. Policy Paradox. The Art of Political Decision Making. New York: W. W. Norton \& Company.

Tilly, Charles. 2008. Why? What happens when people give reasons... and why. Princeton: Princeton University Press.

Viehöver, Willy. 2006. Diskurse als Narrationen. In Handbuch Sozialwissenschaftliche Diskursanalyse. Band 1: Theorien und Methoden, Hrsg. Reiner Keller, Andreas Hierseland, Werner Schneider und Willy Viehöver, 179-208. Wiesbaden: VS.

Viehöver, Willy. 2012. „Menschen lesbar machen“ - Narration, Diskurs, Referenz. In Erzählungen im Öffentlichen. Über die Wirkung narrativer Diskurse, Hrsg. Markus Arnold, Gert Dressel und Willy Viehöver, 65-134. Wiesbaden: Springer VS.

Wagenaar, Hendrik. 2011. Meaning in Action. Interpretation and Dialogue in Policy Analysis. Armonk/London: M. E. Sharpe.

Wendt, Alexander. 1992. Anarchy is what States make of it: The Social Construction of Power Politics. International Organization 46: 391-425.

White, Hayden. 1987. The Content of the Form. Narrative Discourse and Historical Representation. Baltimore/London: The Johns Hopkins University Press.

White, Hayden. 2008. Metahistory. Die historische Einbildungskraft im 19. Jahrhundert in Europa, Frankfurt a. M.: Fischer Taschenbuch. 
Yildiz, Taylan. 2012. Demokratie und Staatstechnik. Eine praxeologische Rekonstruktion von Regime-Hybridität in der Türkei. Baden-Baden: Nomos.

Yildiz, Taylan. 2014. Transitional Justice in der Türkei: Unrechtsdiskurse und kollektive Identitätserzählungen. In Transitional Justice. Aufarbeitung von Unrecht, Rechtsstaatlichkeit und Demokratie, Hrsg. Anja Mihr, Gert Pickel und Susanne Pickel. Wiesbaden: Springer VS (im Erscheinen).

Youtube-Videos: ${ }^{7}$

DutchTurkish. 2011. London Riots: Turkish community attacks the looters. 10.08.2011. http://www.youtube.com/watch?v=XZoGDVOX32U. Zugegriffen: 23.01.2013.

Gaylipooo. 2011. Brave Hero Turks Chase Away Looters !! Street Fight in England 10.08.2011. http://www.youtube.com/watch?v=SIo68T1E38g. Zugegriffen: 23.01.2013.

Genesiselijahtv. 2011. Genesis Elijah - RE: UK Riots. 10.08.2011. http://www.youtube. $\mathrm{com} /$ watch?v=f-rQpkvLuv0\&feature=player_embedded\#!. Zugegriffen: 24.01.2013.

NatCen1. 2012. Reflecting on the riots. 23.07.2012. http://www.youtube.com/ watch?feature=player_embedded $\& v=t$ j4lTxwQfTg. Zugegriffen: 22.01.2013.

Notesproducer. 2011. Tottenham youths reflecting on riots. 12.08.2011. http://www.youtube.com/watch?v=6-XAK6kuoFI. Zugegriffen: 24.01.2013.

nsotd2. 2011. London Riots 2011 Sky News - Teen Gang Members Explosive Interview. 12.08.2011. http://www.youtube.com/watch?v=HjWifAuTwJg. Zugegriffen: 24.01.2013. TheKoshyar. 2011. London, Birmingham, Manchester Riots. Man getting robbed. Disgusting. 08.08.2011. http://www.youtube.com/watch?v=fsRwwQJdKjE. Zugegriffen: 22.01.2013. XItwigyIX. 2011. Speech by Brave Hackney Woman Against London Rioters/ Looters [SUBTITLED] 08.08.2011 http://www.youtube.com/watch?v=ITJcparImeQ. Zugegriffen: 23.01.2013.

7 Da es kaum Standards zur Zitation von Youtube-Videos gibt, schließen wir uns folgendem Stil an: Als Urheber/Verfasser gilt der Name des Users. Das Datum der Veröffentlichung wird übernommen, unabhängig von der Zeit des dargestellten Ereignisses. 


\section{Autorenangaben}

Dr. Frank Gadinger,

Käte Hamburger Kolleg/Centre for Global Cooperation Research,

Universität Duisburg-Essen,

Forschungsprojekt „Politische Narrative“,

Schifferstr. 196,

47059 Duisburg,

gadinger@gcr21.uni-due.de

Sebastian Jarzebski, M.A.,

NRW School of Governance, Universität Duisburg-Essen, Institut für Politikwissenschaft, Forschungsprojekt „Politische Narrative”,

Lotharstr. 53 (Gebäude LS),

47057 Duisburg,

sebastian.jarzebski@uni-due.de

Dr. Taylan Yildiz,

NRW School of Governance, Universität Duisburg-Essen, Institut für Politikwissenschaft, Forschungsprojekt „Politische Narrative“,

Lotharstr. 53 (Gebäude LS),

47057 Duisburg,

taylan.yildiz@uni-due.de 